\title{
Hashimoto's Encephalopathy Presenting as Acute Psychosis
}

\section{Rameshwar Nath Chaurasia* and Vijay Mishra}

Associate Professor, Institute of Medical Sciences, Banaras Hindu University, Neurology, India

\begin{abstract}
Background: Hashimoto's encephalopathy is a relapsing encephalopathy occurring in association with Hashimoto's thyroiditis, with high titers of anti-thyroid antibodies. Clinically the patients may presents with acute or subacute encephalopathy, seizure, myoclonus, and tremulousness, stroke like episode, amnesia or dementia. Here we are reporting a case of hashimoto's encephalopathy who presented with features of acute psychosis.

Case Report: A 56 years old Asian female presented with acute onset of altered behavioural abnormality and declining brain function. Serology revealed high anti thyroperoxidase antibodies. EEG and MRI were consistent with hashimoto's encephalopathy.

Conclusion: Hashimoto's encephalopathy is a rare complication of hashimoto's thyroiditis and is a diagnosis of exclusion and should be suspected in a case of encephalopathy, high anti thyroid antibodies and response to glucocorticoid.
\end{abstract}

Keywords: Hashimoto's encephalopathy; Acute psychosis; Magnetic resonance imaging

\section{Introduction}

Hashimoto's encephalopathy has been described as an encephalopathy, with acute or subacute onset, accompanied by seizures, tremor, myoclonus, ataxia, psychosis, and stroke like episode with relapsing/remitting or progressive course. Hashimoto's encephalopathy is supposed to be of autoimmune origin as supported by its association with other autoimmune diseases. Hashimotos encephalopathy is more common in women than in men. It has been reported in paediatric, adult and elderly populations throughout the world. Hashimoto's encephalopathy is associated with CSF changes, EEG and radiological abnormalities. Hashimoto's encephalopathy appears to be a rare disorder, but, as it is responsive to treatment with corticosteroids, it must be considered in cases of 'investigation negative encephalopathy. Here we report an interesting case of Hashimoto's encephalopathy who presented with psychiatric manifestations.

\section{Case Report}

A 56 years old female diagnosed previously as hypertensive and hypothyroid on 25 microgram of levothyroxine daily presented with features of acute psychosis in the form that she was "surrounded by her enemies" and they were "trying to kill her" and she started abusing them and started biting them. She also had auditory hallucinations and psychomotor agitation. Over a period of three to four days she

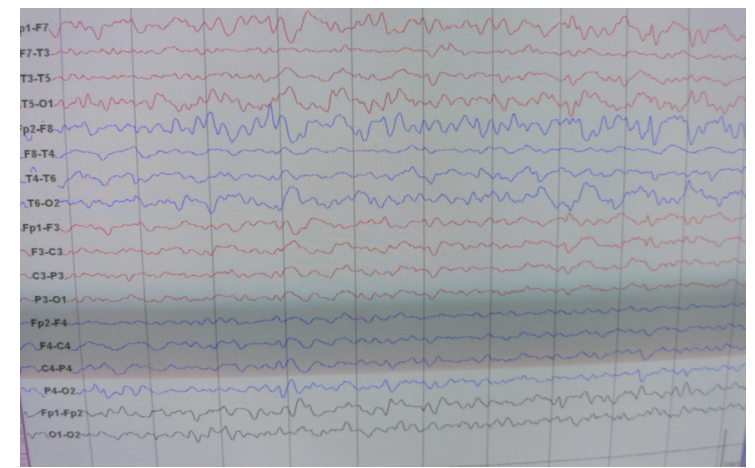

Figure 1: Electroencephalogram showing generalized slow background activity. became irritable, irrelevant talking, aggressive behaviour and urinary incontinence, altered sleep cycle, and later only stuttering speech. Although she did not have fever, headache, vomiting, sensory or motor abnormality or history of previous similar complains or any other psychiatric complains. On examination she was restless, irritable, apraxic, disoriented and not following commands with incomprehensible sound.

Her blood pressure was 160/90 in her right arm supine position, her oral temperature was $38.6 \mathrm{c}$, and respiratory rate was 18 /minute. Pupils were bilateral small and reacting to light and consensual reflex was present. She had no sensory or motor deficit. There was no hepatosplenomegaly and examination of chest and heart were normal.

Routine lab examinations including complete blood count, liver function test and renal function test were within normal parameters. CSF examination showed no cells, sugar of $77 \mathrm{mg} / \mathrm{dl}$ and protein of 40 $\mathrm{mg} / \mathrm{dl}$. Thyroid function test revealed T3, T4 and TSH of $0.77,4.77$ and 10.87 respectively and anti thyroperoxidase antibody value of 2147.50 units. Chest $\mathrm{x}$ ray was within normal limits and two dimensional colour Doppler of heart showed normal heart functions with mild left ventricular hypertrophy. Additionally, antinuclear antibody titre, anti-double-stranded DNA, anti-hepatitis B core antigen, hepatitis B surface antigen, anti-hepatitis $\mathrm{C}$ virus, lupus anticoagulant and Venereal Disease Research Laboratory test results were negative. The electroencephalogram (EEG) showed a slow background activity (Figure1).

T2 weighted MRI brain showed chronic lacunar infarct in bilateral periventricular region (Figure 2). During course of the management of the patient, Injection Methyl prednisolone was given intravenously

*Corresponding author: Rameshwar Nath Chaurasia, Associate Professor Institute of Medical Sciences, Banaras Hindu University, Neurology, Lanka, Varanasi, 221005, India, Tel: +919415353255; Fax: +91-542-2309073; E-mail: goforrameshwar@gmail.com

Received October 18, 2014; Accepted November 27, 2014; Published December 03, 2014

Citation: Chaurasia RN, Mishra V (2014) Hashimoto's Encephalopathy Presenting as Acute Psychosis. Int J Neurorehabilitation 1: 131. doi:10.4172/ijn.1000131

Copyright: (c) 2014 Chaurasia RN, et al. This is an open-access article distributed under the terms of the Creative Commons Attribution License, which permits unrestricted use, distribution, and reproduction in any medium, provided the original author and source are credited. 


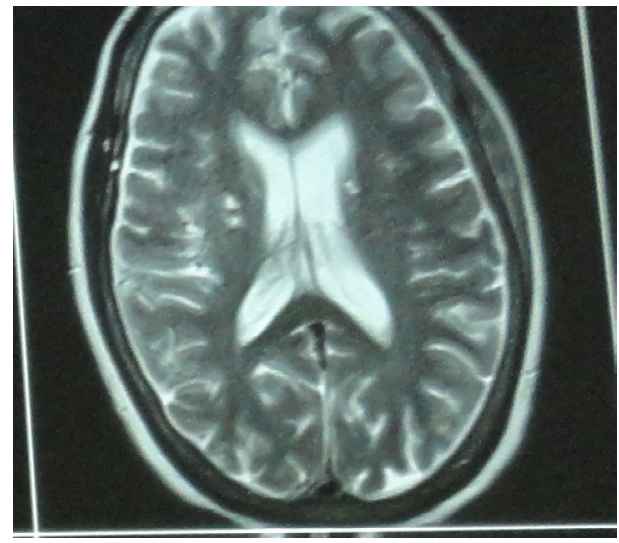

Figure 2: T2W MRI brain image showing bilateral periventricular lacunar ischemic infarct.

for next 5 days, and was followed by oral steroids. On $7^{\text {th }}$ day, patient showed marked improvement of sensorium and by end of $2^{\text {nd }}$ week she started communication through spoken words. The repeat serum autoantibody level was, on day $13^{\text {th }}$ of admission which was declined to 1225 units. Psychosis, which was the major presentation, disappeared by end of $2^{\text {nd }}$ week. , but her symptoms relapsed as we started tapering the steroid after $2^{\text {nd }}$ week. Therefore we started full dose of steroid again for 1 month period then tapered off. She was discharged, after 4 week of hospitalization without antipsychotic drugs, and was fully conscious and aware, was able to carry her activity of daily living.

\section{Discussion}

Hashimoto's encephalopathy in patient with hypothyroidism, who is on replacement therapy, may be precipitated by infection, trauma, burn, or other metabolic stressors. More than a 30 case reports have been published since Professor Brain described it first in 1966 [1]. Clinical manifestations can vary from cognitive impairment and seizure to ataxia and movement disorder. The average age of onset in reported cases [2-10] is 47 years (range, 14 to 78 years). Approximately $85 \%$ of the patients are women. HE can manifest in two major ways: 1) relapsing/ remitting, also referred as vasculitic type, manifesting as encephalopathy and stroke-like episodes. 2) Diffuse progressive typeinsidious onset and progressive course with fluctuations and manifesting with psychiatric symptoms and dementia [11]. Our patient presented with acute psychosis in the form of hallucinations followed by subacute encephalitis. Differential diagnoses includes conditions are Creutzfeldt-Jakob disease, rapidly progressive dementias, and paraneoplastic and non-paraneoplastic limbic encephalitis. With the background history of hypothyroidism and atypical presentation as acute psychosis associated with high titres of antithyroid antibodies, in particular antithyroid peroxidase antibodies followed by subacute encephalitis, we suspected hashimoto's encephalopathy. Thyroid auto antibodies were positive and EEG abnormality was consistent with the diagnosis of hashimoto's encephalopathy which showed persistent fall in levels with clinical improvement.

Pathogenesis of the disease is still not completely defined although autoimmunity is supposed to be the triggering factor [12]. Some findings suggest acute disseminated encephalomyelitis as potential models [6], while others favour cerebral angiitis as a paradigm [5]. This is further supported by the response to immunosuppressive therapy [13] and presence of several neuronal and thyroid auto antibodies [14]. While rapid improvement can be observed within 1 to 3 days, as in the case of our patient improvement started after one week, the average time from start of therapy to significant clinical improvement is 4 to 6 weeks [14]. Neuroradiology studies frequently reveal nonspecific findings, such as bilateral subcortical high signal lesions on T2-weighted images [14], or mild cerebral atrophy with temporal predominance [10]. Similarly findings in our case were nonspecific lacunar infarcts that were most probably due to long standing hypertension. Cerebral angiograms (reported in 10 cases) and Doppler sonograms of cerebral vasculatures (reported in 5 cases) are normal.

To sum up keep high index of suspicion in any patient with thyroid disorder with atypical presentation of acute psychosis and encephalopathy [15] should always be suspected for hashimoto's encephalopathy as it is a treatable condition and would avoid mismanagement and unnecessary medications and is very rewarding.

\section{References}

1. Brain L, Jellinek EH, Ball K (1966) Hashimoto's disease and encephalopathy. Lancet 2: 512-514.

2. Thrush DC, Boddie HG (1974) Episodic encephalopathy associated with thyroid disorders. J Neurol Neurosurg Psychiatry 37: 696-700.

3. Mauriac L, Roger P, Kern AM (1982) Thyroidite de Hashimoto et encephalopathie. Rev Franc Endocrinol Clin 23: 147-150.

4. Latinville D, Bernardi O, Cougoule JP, Bioulac B, Henry P, et al. (1985) [Hashimoto's thyroiditis and myoclonic encephalopathy. Pathogenic hypothesis]. Rev Neurol (Paris) 141: 55-58.

5. Shein M, Apter A, Dickerman Z, Tyano S, Gadoth N (1986) Encephalopathy in compensated Hashimoto thyroiditis: a clinical expression of autoimmune cerebral vasculitis. Brain Dev 8: 60-64.

6. Henderson LM, Behan PO, Aarli J (1987) Hashimoto's encephalopathy: a new neuroimmunological syndrome. Ann Neurol 22: 140-141.

7. Shaw PJ, Walls TJ, Newman PK, Cleland PG, Cartlidge NE (1991) Hashimoto's encephalopathy: a steroid-responsive disorder associated with high anti-thyroid antibody titers--report of 5 cases. Neurology 41: 228-233.

8. Ghawche F, Bordet R, Destée A (1992) [Hashimoto's encephalopathy: toxic or autoimmune mechanism?]. Rev Neurol (Paris) 148: 371-373.

9. Henchey R, Cibula J, Helveston W, Malone J, Gilmore RL (1995) Electroencephalographic findings in Hashimoto's encephalopathy. Neurology 45: 977-981.

10. Ghika-Schmid F, Ghika J, Regli F, Dworak N, Bogousslavsky J, et al. (1996) Hashimoto's myoclonic encephalopathy: an underdiagnosed treatable condition? Mov Disord 11: 555-562.

11. Fatourechi V (2005) Hashimoto's encephalopathy: myth or reality? An endocrinologist's perspective. Best Pract Res Clin Endocrinol Metab 19: 53-66.

12. Chong JY, Rowland LP, Utiger RD (2003) Hashimoto encephalopathy: syndrome or myth? Arch Neurol 60: 164-171.

13. Marshall GA, Doyle JJ (2006) Long-term treatment of Hashimoto's encephalopathy. J Neuropsychiatry Clin Neurosci 18: 14-20.

14. Kothbauer-Margreiter I, Sturzenegger M, Komor J, Baumgartner R, Hess CW (1996) Encephalopathy associated with Hashimoto thyroiditis: diagnosis and treatment. J Neurol 243: 585-593.

15. Gómez-Bernal GJ, Reboreda A, Romero F, Bernal MM, Gómez F (2007) A Case of Hashimoto's Encephalopathy Manifesting as Psychosis Prim Care Companion. J Clin Psychiatry 9: 318-319. 